\title{
Performance Enhancement of Diindenoperylene-based Organic Photovoltaic Cells by Nanocolumn-Arrays
}

\author{
Shuwen Yu, ${ }^{1}$ Andreas Opitz, ${ }^{1, *}$ Stefan Grob, ${ }^{2}$ Roland Resel, ${ }^{3}$ Martin Oehzelt,,${ }^{1,4}$ \\ Wolfgang Brütting, ${ }^{2}$ Ingo Salzmann, ${ }^{1}$ and Norbert Koch $^{1,4, *}$ \\ ${ }^{1}$ Humboldt-Universität zu Berlin, Institut für Physik \& IRIS Adlershof, Brook-Taylor Straße 6, \\ 12489 Berlin, Germany \\ ${ }^{2}$ Augsburg University, Institut für Physik, Universitätsstraße 1, 86135 Augsburg, Germany \\ ${ }^{3}$ Graz University of Technology, Institute of Solid State Physics, Petersgasse 16, 8010 Graz, \\ Austria \\ ${ }^{4}$ Helmholtz-Zentrum für Materialien und Energie GmbH, Bereich Solarenergieforschung, \\ Albert-Einstein-Straße 15, 12489 Berlin, Germany \\ E-mail addresses: Andreas.Opitz@hu-berlin.de (A. Opitz) \\ Norbert.Koch@physik.hu-berlin.de (N. Koch)
}

\begin{abstract}
Crystalline and uniform nanocolumns of the organic semiconductor diindenoperylene (DIP) were fabricated by glancing-angle deposition and employed in organic photovoltaic cells (OPVCs) forming an interdigitated donor/acceptor heterojunction, with fullerene as electron acceptor. In comparison to reference bilayer devices the nanocolumn-based solar cells exhibit increased power conversion efficiency. Based on a comprehensive structural and morphological analysis, we identify three advantages of the interdigitated nanocolumn structures: (i) The active donor/acceptor interface area, crucial for exciton dissociation, is increased and the column diameter is in the range of the exciton diffusion length. (ii) The molecular orientation of DIP is such in the nanocolumns that light absorption is enhanced. (iii) The ubiquitous presence of vertical interfaces throughout nanocolumn-based devices is further beneficial to light absorption, as it fully compensates wavelength-dependent interference effects within the device structure. This work shows how the benefits of nanocolumns can go beyond simple interface area enlargement to improve the efficiency of OPVCs.
\end{abstract}




\section{Introduction}

Since the initial application of "sandwich" structure in organic photovoltaic cells (OPVCs) in the 1970s, ${ }^{1,2}$ the power conversion efficiency of OPVCs significantly increased only after introducing the donor/acceptor heterojunction concept, ${ }^{3}$ where layers of preferentially hole/electron conducting materials with an interface promoting exciton dissociation are employed. Today, high open circuit voltage $\left(V_{o c}\right)$ and optimized light harvesting can be realized with a wide variety of organic semiconductors suitable for application in OPVCs. ${ }^{4-13}$ However, the short diffusion length of photo-generated excitons in such materials (typically in the range of tens of nanometers ${ }^{14,}{ }^{15}$ ) significantly limits the fraction of excitons dissociated at the donor/acceptor interface, thus restricting the generation of mobile charges. To reconcile the exciton diffusion length with the average dimensions of organic heterojunctions, the concept of bulk heterojunction was introduced, which allows increasing the effective area of the donor/acceptor interface and, simultaneously, reducing the separation between individual donor and acceptor volumes. ${ }^{16-18}$ While this approach turned out to be highly successful for polymer-based OPVCs processed from solution ${ }^{19}$ and molecular blend structure OPVCs typically from vacuum co-deposition ${ }^{10,}$ 20, 21, low charge-carrier mobility, exciton quenching, and morphological issues like dead-ends in the conduction pathway (i.e., individual, isolated phase grains without connection to the respective electrode) still limit the desired efficiency improvement of this approach ${ }^{22-24}$. Idealized structures to overcome these limitations are bulk heterojunction OPVCs comprising a crystalline, vertically interdigitated, and laterally structured configuration of separate donor/acceptor phases, with the aim to enable generated excitons to most likely reach an interface and the separated charges to be efficiently collected by the electrodes through individual donor/acceptor zones. ${ }^{25,26}$ As demonstrated in previous studies, a preparation technique highly promising for realizing such structures is glancing angle deposition (GLAD), ${ }^{27-30}$ as schematically illustrated in Fig. 1a, where high aspect-ratio nanocolumns (NCs) are established through shadowing effects. ${ }^{25,26,31,32}$ 
Here, we compare OPVCs based on a conventional bilayer heterojunction of a donor/acceptor pair to devices with interdigitated heterojunctions comprising NC arrays. Diindenoperylene (DIP) was chosen as donor material to establish NC arrays via GLAD as it is an emerging material for OPVCs ${ }^{13}$. To accomplish the interdigitated donor/acceptor heterojunction, fullerene (C60) was employed as acceptor material by subsequent (vertical) physical vapor deposition in order to fill the voids between the NCs, as schematically illustrated in Fig. 1b. We show that the performance of NC-based devices is significantly improved compared to the bilayer reference OPVCs, which we relate to the increase in active interface area and enhanced absorption of the NC arrays, as well as reducing the influence of the "trade-off' between getting high light absorption with thick C60 films and obtaining good exciton dissociation efficiency, which requires a film thickness close to the exciton diffusion length.

\section{Experimental}

ITO coated glass substrates (Präzisions Glas \& Optik, sheet resistance $<20 \Omega /$ sq, surface roughness (rms): $2 \mathrm{~nm}$ ) were sonicated for $10 \mathrm{~min}$ in acetone and, subsequently, isopropanol. UV-ozone-treatment was applied to improve the wetting of the substrate by an aqueous suspension of the intrinsically conducting polymer poly(ethylene-dioxythiophene):poly(styrenesulfonate) (PEDOT:PSS) (H.C. Starck GmbH, HIL 1.3), employed as substrate for OPVCs. PEDOT:PSS was spin-coated and subsequently annealed at $200^{\circ} \mathrm{C}$ for 5 min under ambient conditions to desorb residual water. DIP was purified twice by gradient sublimation. NCs were grown by GLAD, the material sublimed from a resistively heated ceramic crucible with a deposition rate of ca. $0.1 \mathrm{~nm} / \mathrm{s}$ (quartz crystal microbalance, base pressure $<5 \times 10^{-7}$ mbar) at room temperature. The incident angle of the molecular flux $(\alpha)$ with respect to the substrate surface normal was set to $84^{\circ}$ (c.f. Fig. 1a) and the substrate rotation frequency $(\omega)$ was 6 rounds per minute (computer-controlled step-motor). OPVCs were completed by $75 \mathrm{~nm}$ thick C60 (99.9\%, Sigma-Aldrich) deposited at a rate of 
$0.02 \mathrm{~nm} / \mathrm{s}$ on the DIP films/NCs in another different vacuum chamber (base pressure $<2 \times 10^{-8}$ mbar); sample transfer between the chambers was done through ambient. A $6.5 \mathrm{~nm}$ thick bathocuproine (BCP, 96\%, Sigma-Aldrich) layer was deposited onto the donor/acceptor structure followed by a $100 \mathrm{~nm}$ thick samarium $(\mathrm{Sm})$ layer used as cathode. The current density versus voltage $(\mathrm{J}-\mathrm{V})$ characteristics were measured in-situ in dark and under illumination $\left(25 \mathrm{~mW} / \mathrm{cm}^{2}\right.$, Solux C6 halogen lamp, close to daylight spectrum in the visible region) with a Keithley 2400 SourceMeter $^{\circledR}$. The sample morphology was investigated with a Bruker Multimode-8 atomic force microscope (AFM) and a HITACHI S-4100 field-emission scanning electron microscope (SEM), using the latter after sputter-coating the samples with a thin gold layer to avoid sample charging. Optical absorption spectra (UV-VIS) were recorded with a PerkinElmer Lambda 900 spectrometer. X-ray diffraction experiments were carried out in inert $(\mathrm{He})$ atmosphere at beamline $\mathrm{W} 1$ at the synchrotron radiation source HASYLAB (DESY, Germany) using a primary beam energy of $10.5 \mathrm{keV}$ and a one-dimensional position sensitive detector (Mythen 1K, Dectris) mounted in z-direction covering a range of out-of-plane scattering angles of ca. $3.8^{\circ}$; for grazing-incidence diffraction, five scans at fixed out-of-plane position were performed for every reciprocal space map ${ }^{33}$; specular X-ray diffraction on the PEDOT:PSS-substrate samples was performed with a Philips X'Pert system in Bragg-Berntano geometry (sealed Cr-tube, secondary HOPG monochromator).

\section{Results and Discussion}

\section{A. Morphology}

The formation of individual NCs upon GLAD is due to limited adsorbate diffusion, shadowing effects of the collimated molecular flux under oblique angle $\left(\alpha>70^{\circ}\right)$, and continuous rotation of the substrate (c.f. Fig. 1a), which leads to a large fraction of the incoming flux being captured by larger grains that, therefore, preferentially grow. ${ }^{28,32,34-37}$ Fig. 2 illustrates the representative topography and cross section of conventional vertically deposited DIP films on bare (a) and PEDOT:PSS 
pre-covered ITO (b), as well as DIP-NCs grown by GLAD on the pristine (c), and the polymer-coated substrates (d). The vertically deposited film on bare ITO (Fig. 2a) shows a morphology of (terraced) islands as expected for DIP. ${ }^{38,39}$ In contrast, with GLAD a highly corrugated morphology of polygon-shaped nanocolumns reaching down to the ITO substrate is apparent (Fig. 2c). As a result, the surface roughness (root-mean-square-rms: $9.2 \mathrm{~nm}$ ) is increased by more than a factor of two (rms: 22.4 $\mathrm{nm})$ through GLAD, and the bottom radius of the NCs is in the range between 60 and $80 \mathrm{~nm}$ (c.f. Fig. 2c); this is well within the range of the reported exciton diffusion length of crystalline DIP between 20 and $100 \mathrm{~nm}^{15,40}$. In contrast, DIP-NCs on the PEDOT:PSS coated ITO substrates exhibited some connectivity and had a broader distribution of diameters (Fig. 2d), which is a direct result of the increased molecular diffusibility on the smoother surface. To enhance the shadow effect, a $10 \mathrm{~nm}$ thick DIP film was vertically deposited on the substrates prior to GLAD to increase the substrate roughness, in analogy to a procedure introduced in a previous study on pentacene $\mathrm{NCs}^{32}$. With this approach, the homogeneity of the NCs on the bare ITO substrate was significantly improved (Fig. 2e) and essentially monodisperse columns were formed with mean lateral and vertical extensions of $65 \mathrm{~nm}$ (radius) and $120 \mathrm{~nm}$, respectively. For the PEDOT:PSS covered ITO substrates, basically the same trend was observed, however, with an almost doubled void area between the NCs (Fig. 2f). Finally, the donor/acceptor heterostructures were completed by vertical vacuum deposition of nominally $75 \mathrm{~nm}$ C60 on both the vertically deposited DIP film (denoted as planar in the following) and the DIP-NC/DIP (columnar); the topography and the respective cross section micrographs (obtained by SEM) are shown in Fig. 3a-d. The comparison with the DIP-only samples reveals that fullerene molecules completely cover the vertically deposited DIP layer (Fig. 3a-b) and also fully penetrate into the voids between the NCs (Fig. 3c-d). For the NCs, this is clearly seen in Fig. 3d from the absence of a clear-cut DIP/C60 interface, which is, in contrast, clearly observed in the cross section view of the bilayer structure (Fig. 3b). Finally, the AFM micrographs shown in Fig. 4 were used to determine the surface-area enlargement factor $(\xi)$ of the 
films, which is increased from $\xi=1.068$ for planar film to $\xi=1.288$ for NC structures made by GLAD; note that this value represents a lower limit for $\xi$ due to the finite curvature of the AFM tip.

\section{B. Structure}

To assess the microstructure of our samples we performed grazing-incidence X-ray diffraction (GIXRD) and specular X-ray diffraction (XRD). Figure 5a shows GIXRD in-plane data of C60 on vertically deposited DIP (black curve) compared to C60 on DIP-NCs (red curve) and two reference scans on DIP-NCs (blue curve) and pristine C60 (black curve), all deposited on ITO (c.f. Fig. 2 for respective SEM data). In all cases, DIP is found to grow crystalline in two different polymorphs with the dominant one denoted as $B$ ("bulk phase") and the minor one as $B$ ' in the following. In the corresponding specular XRD scans (Fig. 6) only one peak series is observed with a (001)-lattice spacing of $1.665 \mathrm{~nm}$, which is indicative of an almost upright-standing molecular orientation. Via the corresponding reciprocal space maps (Fig. 6), both observed polymorphs $B$ and $B^{\prime}$ are identified to be significantly different from known crystal structures, that is, single-crystal ${ }^{41}$ and thin-film phases ${ }^{42}$, the latter typically denoted as $\sigma-, \lambda$, and $\lambda^{*}$-phases in literature $39,43,44$. An unambiguous determination of the unit-cell parameters is impossible in the present case, as the diffraction intensities are smeared out along rings in the reciprocal space maps. However, combining our GIXRD and specular XRD results, the unit-cell dimensions can be roughly estimated, if, in analogy with known DIP crystal structures 41,38 , a monoclinic space-group with a monoclinic angle of $\beta=92.4^{\circ}$ is assumed. Taking into account the $(11 l),(02 l),(12 l)$, and (13l) reflections, this assumption yields unit-cells of $a=0.708 \mathrm{~nm}$ and $a^{\prime}=0.642 \mathrm{~nm}, b=b^{\prime}=0.846 \mathrm{~nm} c=c^{\prime}=1.666 \mathrm{~nm}$ for the $B$ - and $B^{\prime}$-phase, respectively. We note that, while the $B^{\prime}$-polymorph seems to be a yet unreported crystal structure likely mediated by the substrate, the small deviation of the $B$-phase lattice parameters from the single-crystal solution might be due to thermal expansion ${ }^{41}$. While the in-plane reflections in the $q_{\|}$-range between $1-2 \AA^{-1}$ 
due to DIP molecules in upright orientation are present in all DIP samples, both samples comprising DIP-NCs further show the (001)-reflection also in GIXRD, which is indicative of additional crystalline grains in a "lying" molecular orientation. Therefore, the vertically deposited film can be regarded as fiber-textured (“2D-powder"), DIP-NCs show a more "3D-powder like" crystalline property. This can be best seen in the full reciprocal space maps (Fig. 6), where the DIP-related peaks of the NC samples are smeared out significantly more than those of the vertically deposited film. In contrast to DIP on smooth substrates like $\mathrm{SiO}_{\mathrm{x}}{ }^{45}$, such rings are also observed for vertically deposited DIP on ITO, which is attributed to the higher surface roughness of this substrate that increases the mosaicity of the adsorbate. Finally, the C60 top-layer is found to grow crystalline on both types of DIP underlayers, which is in line with previous findings for $\mathrm{C} 60 / \mathrm{DIP} / \mathrm{SiO}_{\mathrm{x}}{ }^{46}$ or C60/pentacene ${ }^{22}$.

Both for vertically deposited DIP and the DIP-NCs, a similar growth behavior is found on PEDOT:PSS coated ITO substrates, as illustrated by the XRD data depicted in Figure 5b. Again, the DIP $(00 l)$-series with an identical lattice spacing of $1.66 \mathrm{~nm}$ is found, but in contrast to bare ITO substrates, now up to higher order owing to the lower surface roughness of the polymer coating. At $1.47 \AA^{-1}$ we observe an additional reflection related to lying DIP, which was denoted as $\lambda$-phase in previous studies ${ }^{45}$. Compared to the vertically deposited DIP film with a peak ratio of 9:1 (area) between the (004) and the $\lambda$-phase peak, this ratio changes to almost 1:1 for DIP NCs (see inset in Fig. 5b), thus, clearly indicating - in analogy to above data on the pristine ITO substrates - an increase of the lying DIP fraction in the samples grown by GLAD.

\section{Photovoltaic Cells}

We now turn to employing these structures in OPVCs in order to assess the impact of NCs on the device performance. We compare OPVCs of planar and NC form, both capped with a $6.5 \mathrm{~nm}$ BCP layer followed by a $100 \mathrm{~nm} \mathrm{Sm} \mathrm{top-electrode.}$ 
Note that the beneficial role of BCP in such device structures has been intensively investigated and was attributed to protection of the active layer during metal deposition. ${ }^{47,} 48$ The J-V characteristics of both devices were recorded under 25 $\mathrm{mW} / \mathrm{cm}^{2}$ illumination from a halogen lamp in vacuum, the results are shown in Fig. $7 \mathrm{a}$ with the corresponding key parameters summarized in Table 1. While the open circuit voltage $\left(V_{o c}\right)$ of both OPVCs is almost identical (ca. $\left.0.8 \mathrm{~V}\right)$, the short-circuit current $\left(J_{s c}\right)$ under illumination is almost $20 \%$ higher in the NC-based OPVCs. Furthermore, the current density decreases less pronounced in the fourth quadrant for the NC devices, because the series resistance $\left(R_{S}\right)$ is lower by more than one order of magnitude, which further explains the higher fill factor (FF). ${ }^{49}$ We attribute the tremendously smaller $R_{S}$ for the NC-based OPVCs to closed pathways within the active donor/acceptor layer. Moreover, the higher FF is further related to the lower ideality factor $(n)$ of the NC-OPVCs $(n=1.1)$ compared to that that of the bilayer OPVCs $(n=6.4)$. These values were deduced from the slope of the logarithmically represented $\mathrm{J}-\mathrm{V}$ characteristics in the exponential regime (inset in Fig. 7a) based on the non-ideality diode equation: ${ }^{49}$

$$
J=J_{0}\left\{\exp \left[e\left(V-J R_{S}\right) / n k T\right]-1\right\}
$$

where $J_{0}$ is the saturation current density, $n$ the diode ideality factor, $k$ Boltzmann's constant and $T$ the temperature.

To assess the influence of air exposure, e.g., due to sample transfer through air or poor encapsulation, reference OPVCs were fabricated and characterized in-situ under conditions of simulated AM 1.5 illumination at $100 \mathrm{~mW} / \mathrm{cm}^{2}$ (Fig. 7b). Compared with the performance of the air-exposed OPVCs, almost identical J-V characteristics are observed, which well documents the stability of the DIP-NCs against (short) ambient exposure.

Since the DIP structure and molecular orientation varied between planar and NC devices, we compare the absorption characteristics of DIP of corresponding samples in Fig. 8. On average, a $50 \mathrm{~nm}$ planar film absorbs 1.5 more photons than the NC 
structure (10 nm underlayer plus $100 \mathrm{~nm}$ columns), but clearly, due to the inherent structural differences, their absorption spectra cannot be directly compared. Therefore, individual samples of both types were fully dissolved in a defined volume of 1,2-dichlorobenezene to quantify the total amount of DIP contained therein. We found that the volume of DIP present in the NC-OPVCs equals that of a $20 \mathrm{~nm}$ thick DIP film fabricated by vertical deposition; the inset in Fig. 8 depicts a comparison of the respective solution spectra. From these data it is apparent that the NC sample exhibits a significantly higher absorbance than the vertically deposited sample (normalized to the number of molecules present in each sample). This finding can be understood by the different molecular orientation of DIP in the planar film and in NCs. Planar films comprise a fiber-textured with a preferential vertical molecular orientation ("2D-powder"), while the NCs exhibit significantly less-textured growth (more "3D-powder like") and a substantial fraction of "lying" molecules. As the absorption is dominated by the fundamental HOMO-LUMO transition (c.f. inset in Fig. 8), which is polarized along the long molecular axis of DIP, this transition is less efficiently excited in the vertically deposited film than in the DIP-NCs. ${ }^{50}$

Overall, with less DIP molecules (factor 0.4, as deduced from UV-VIS) and, therefore, lower total absorption by DIP NCs (c.f. Fig. 8), the power conversion efficiency $(\eta)$ of $\mathrm{NC}-\mathrm{OPVC}$ reaches $1.8 \%$, significantly larger than that of the stronger absorbing planar structure (bilayer OPVC, $\eta=1.4 \%$ ), as listed in Table 1 (Note: The same measurement conditions are utilized for both structures without using a solar simulator. Without spectral corrections the efficiency is in the range as reported in the literature for similar devices ${ }^{20,51,52}$ ). In comparison to the bilayer OPVCs, more photo-generated excitons in NC-OPVCs can reach the donor/acceptor interface for dissociation. This is related to the increase of active interface area in the NC heterojunction by ca. $20 \%$ compared to the planar heterojunction. This is less than the overall efficiency increase of almost 30\%, therefore another mechanism may contribute beneficially. The intrinsic vertical distribution of the donor/acceptor interface introduced through the NCs covers a wider range of high optical intensity, 
which may be generated by interference effects through reflection at the back electrode, ${ }^{14,15,18,20,23}$ reducing the impact of comparably short exciton diffusion length in C60 while employing a thicker film. Moreover, the lying DIP phases may positively influence $\mathrm{J}_{\mathrm{sc}}$ and $\mathrm{FF}$, but due to the unknown spatial distribution of the two DIP orientations we cannot quantify the contribution.

\section{Conclusion}

The morphology and structure of crystalline DIP nanocolumns grown by GLAD on pristine and PEDOT:PSS coated ITO substrates was revealed and such structures applied in OPVCs. Despite the fact that the DIP volume in NC-based OPVCs was less than one half of that in planar heterojunctions (with also an overall lower absorption by the NCs), the power-conversion efficiency of the solar cells is increased from $1.4 \%$ (planar) to $1.8 \%$ through employing DIP-NCs in the active layer. This noteworthy finding is explained by (i) a $20 \%$ increase of active interface area between DIP-NCs and the surrounding C60 acceptors, which improves charge-carrier generation through exciton dissociation at that interface. (ii) A more random orientation of crystalline DIP grains in the NCs enhances light absorption in comparison to the fiber-textured, upright standing DIP in vertically deposited films. (iii) The vertically oriented donor/acceptor interface enabled by the NCs is further beneficial for light absorption, as it covers a wider vertical range, and light intensity variations through interference effects can be compensated for. The present work underlines the key role of structure and morphology in OPVCs and demonstrates that their targeted optimization by glancing angle deposition offers good perspectives for pushing further the limits of organic photovoltaics in future applications.

\section{Acknowledgements}

We thank Carola Klimm (Helmholtz-Zentrum Berlin, Institut für Silizium Photovoltaik) for help with SEM measurements, Prof. Jürgen P. Rabe (HU-Berlin) for 
granting access to AFM, Ramona Köppl, Harald Thommesen (TU-Graz) and Wolfgang Caliebe (DESY, HASYLAB) for experimental support. This work was supported by the German Research Foundation (DFG) within the priority program SPP 1355 "Elementary Processes of Organic Solar Cells". S.G. thanks the Bavarian Research Foundation (BFS) for funding.

\section{References}

1. C. W. Tang and A. C. Albrecht, J. Chem. Phys. 62 (6), 2139 (1975).

2. A. K. Ghosh and T. Feng, J. Appl. Phys. 44 (6), 2781 (1973).

3. C. W. Tang, Appl. Phys. Lett. 48 (2), 183 (1986).

4. M. Svensson, F. Zhang, S. Veenstra, W. Verhees, J. Hummelen, J. Kroon, O. Inganä and M. Andersson, Adv. Mater. 15, 988 (2003).

5. T. Kietzke, D. A. M. Egbe, H.-H. Horhöld and D. Neher, Macromolecules 39, 4018 (2006).

6. F. Zhang, W. Mammo, L. M. Andersson, S. Admassie, M. R. Andersson and O. Inganäs, Adv. Mater. 18 (16), 2169 (2006).

7. R. F. Bailey-Salzman, B. P. Rand and S. R. Forrest, Appl. Phys. Lett. 91 (1), 013508 (2007).

8. M. Riede, T. Mueller, W. Tress, R. Schueppel and K. Leo, Nanotechnology 19 (42), 424001 (2008).

9. J. C. Bijleveld, R. A. M. Verstrijden, M. M. Wienk and R. A. J. Janssen, Appl. Phys. Lett. 97 (7), 073304 (2010).

10. J. Wagner, M. Gruber, A. Wilke, Y. Tanaka, K. Topczak, A. Steindamm, U. Hörmann, A. Opitz, Y. Nakayama, H. Ishii, J. Pflaum, N. Koch and W. Brütting, J. Appl. Phys. 111 (5), 054509 (2012).

11. L. Dou, J. You, Z. Hong, Z. Xu, G. Li, R. A. Street and Y. Yang, Adv. Mater. 25 (46), 6642 (2013).

12. U. Hörmann, J. Wagner, M. Gruber, A. Opitz and W. Brütting, Phys. Stat. Sol. -R 5 (7), 241 (2011).

13. J. Wagner, M. Gruber, A. Hinderhofer, A. Wilke, B. Bröker, J. Frisch, P. Amsalem, A. Vollmer, A. Opitz, N. Koch, F. Schreiber and W. Brütting, Adv. Funct. Mater. 20 (24), 4295 (2010).

14. T. Stübinger and W. Brütting, J. Appl. Phys. 90 (7), 3632 (2001).

15. D. Kurrle and J. Pflaum, Appl. Phys. Lett. 92 (13), 133306 (2008).

16. G. Yu, J. Gao, J. C. Hummelen, F. Wudl and A. J. Heeger, Science 270 (5243), 1789 (1995).

17. J. J. M. Halls, C. A. Walsh, N. C. Greenham, E. A. Marseglia, R. H. Friend, S. C. Moratti and A. B. Holmes, Nature 376, 498 (2002).

18. P. Peumans, S. Uchida and S. R. Forrest, Nature 425, 158 (2003).

19. W. Ma, C. Yang, X. Gong, K. Lee and A. J. Heeger, Adv. Funct. Mater. 15 (10), 1617 (2005).

20. M. Gruber, M. Rawolle, J. Wagner, D. Magerl, U. Hörmann, J. Perlich, S. V. Roth, A. Opitz, F. Schreiber, P. Müller-Buschbaum and W. Brütting, Adv. Energy Mater. 3 (8), 1075 (2013).

21. X. Xiao, J. D. Zimmerman, B. E. Lassiter, K. J. Bergemann and S. R. Forrest, Appl. Phys. Lett. 102 (7) (2013).

22. I. Salzmann, S. Duhm, R. Opitz, R. L. Johnson, J. P. Rabe and N. Koch, J. Appl. Phys. 104 (11) (2008). 
23. A. Opitz, J. Wagner, W. Brütting, I. Salzmann, N. Koch, J. Manara, J. Pflaum, A. Hinderhofer and F. Schreiber, J. Select. Topics Quantum Electron. 16, 1707 (2010).

24. M. Gruber, J. Wagner, K. Klein, U. Hörmann, A. Opitz, M. Stutzmann and W. Brütting, Adv. Energy Mater. 2 (9), 1100 (2012).

25. F. Yang, M. Shtein and S. R. Forrest, Nature Mater. 4 (1), 37 (2004).

26. N. Koch, Chemphyschem : a Eur. J. Chem. Phys. \& Phys. Chem. 8 (10), 1438 (2007).

27. K. Robbie, M. J. Brett and A. Lakhtakia, Nature Mater. 384 (6610) (1996).

28. K. Robbie and M. J. Brett, J. Vac. Sci. Technol. A 15 (3), 1460 (1997).

29. J. G. Van Dijken, M. D. Fleischauer and M. J. Brett, Org. Electron. 12 (12), 2111 (2011).

30. Y. Zhou, T. Taima, T. Miyadera, T. Yamanari, M. Kitamura, K. Nakatsu and Y. Yoshida, Nano Lett. 12 (8), 4146 (2012).

31. Y. Zheng, R. Bekele, J. M. Ouyang and J. G. Xue, Org. Electron. 10 (8), 1621 (2009).

32. S. W. Yu, C. Klimm, P. Schäfer, J. P. Rabe, B. Rech and N. Koch, Org. Electron. 12 (12), 2180 (2011).

33. I. Salzmann, A. Moser, M. Oehzelt, T. Breuer, X. Feng, Z.-Y. Juang, D. Nabok, R. G. Della Valle, S. Duhm, G. Heimel, A. Brillante, E. Venuti, I. Bilotti, C. Christodoulou, J. Frisch, P. Puschnig, C. Draxl, G. Witte, K. Müllen and N. Koch, ACS Nano 6 (12), 10874 (2012).

34. J. Zhang, I. Salzmann, S. Rogaschewski, J. P. Rabe, N. Koch, F. Zhang and Z. Xu, Appl. Phys. Lett. 90 (19), 193117 (2007).

35. L. Abelmann and C. Lodder, Thin Solid Films 305, 1 (1997).

36. Y. P. Zhao, D. X. Ye, G. C. Wang and T. M. Lu, Nano lett. 2, 351 (2002).

37. J. G. Van Dijken, M. D. Fleischauer and M. J. Brett, J. Mater. Chem. 21 (4), 1013 (2011).

38. A. Turak, M. Nguyen, F. Maye, J. Heidkamp, P. Lienerth, J. Wrachtrup and H. Dosch, J. Nano Res. 14 (2011).

39. A. C. Dürr, F. Schreiber, M. Münch, N. Karl, B. Krause, V. Kruppa and H. Dosch, Appl. Phys. Lett. 81 (12), 2276 (2002).

40. R. R. Lunt, N. C. Giebink, A. A. Belak, J. B. Benziger and S. R. Forrest, J. Appl. Phys. 105 (5) (2009).

41. M. A. Heinrich, J. Pflaum, A. K. Tripathi, W. Frey, M. L. Steigerwald and T. Siegrist, J. Phys. Chem. C 111 (51), 18878 (2007).

42. A. C. Dürr, F. Schreiber, M. Kelsch, H. D. Carstanjen, H. Dosch and O. H. Seeck, J. Appl. Phys. 93 (9), 5201 (2003).

43. S. Kowarik, A. Gerlach, S. Sellner, L. Cavalcanti, O. Konovalov and F. Schreiber, Appl. Phys. A 95 (1), 233 (2009).

44. A. C. Dürr, N. Koch, M. Kelsch, A. Rühm, J. Ghijsen, R. L. Johnson, J. J. Pireaux, J. Schwartz, F. Schreiber, H. Dosch and A. Kahn, Phys. Rev. B 68 (11), 115428 (2003).

45. S. Kowarik, A. Gerlach, S. Sellner, F. Schreiber, L. Cavalcanti and O. Konovalov, Phys. Rev. Lett. 96 (12), 125504 (2006).

46. A. Hinderhofer, A. Gerlach, K. Broch, T. Hosokai, K. Yonezawa, K. Kato, S. Kera, N. Ueno and F. Schreiber, J. Phys. Chem. C 117 (2), 1053 (2012).

47. H. Gommans, B. Verreet, B. P. Rand, R. Muller, J. Poortmans, P. Heremans and J. Genoe, Adv. Funct. Mater. 18 (22), 3686 (2008).

48. M. Vogel, S. Doka, C. Breyer, M. C. Lux-Steiner and K. Fostiropoulos, Appl. Phys. Lett. 89 (16), 163501 (2006). 
49. S. M. Sze, Physics of Semiconductor Devices, 2nd ed. (Wiley, New York, 1981).

50. U. Heinemeyer, R. Scholz, L. Gisslén, M. Alonso, J. Ossó, M. Garriga, A. Hinderhofer, M. Kytka, S. Kowarik, A. Gerlach and F. Schreiber, Phys. Rev. B 78 (8) (2008).

51. A. Steindamm, M. Brendel, A. K. Topczak and J. Pflaum, Appl. Phys. Lett. 101 (14) (2012).

52. C. Schuenemann, A. Petrich, R. Schulze, D. Wynands, J. Meiss, M. P. Hein, J. Jankowski, C. Elschner, J. Alex, M. Hummert, K.-J. Eichhorn, K. Leo and M. Riede, Org. Electron. 14 (7), 1704 (2013).

Table 1: Key parameters of OPVC performance, as deduced from the J-V curves (c.f. Fig 5); planar denotes the bilayer OPVC, column the DIP-NC based device; $V_{O C}$ the open-circuit voltage, $J_{S C}$ the short-circuit current, $F F$ the fill factor, $R_{S}$ the serial resistance, $R_{s h}$ the shunt resistance, and $\eta$ the external power-conversion efficiency under $25 \mathrm{~mW} / \mathrm{cm}^{2}$ illumination (halogen lamp) in vacuum.

\begin{tabular}{ccccccc}
\hline & $\mathbf{V}_{\mathbf{o c}}(\mathrm{V})$ & $\mathbf{J}_{\mathbf{s c}}\left(\mathrm{mA} / \mathrm{cm}^{2}\right)$ & $\mathbf{F F}$ & $\mathbf{R}_{\mathbf{s}}(\Omega)$ & $\mathbf{R}_{\text {sh }}(\mathrm{M} \Omega)$ & $\boldsymbol{\eta}$ \\
\hline planar & 0.79 & 1.05 & 0.43 & 6100 & 0.34 & $1.4 \% \pm 0.05 \%$ \\
column & 0.77 & 1.25 & 0.48 & 260 & 0.17 & $1.8 \% \pm 0.05 \%$ \\
\hline
\end{tabular}




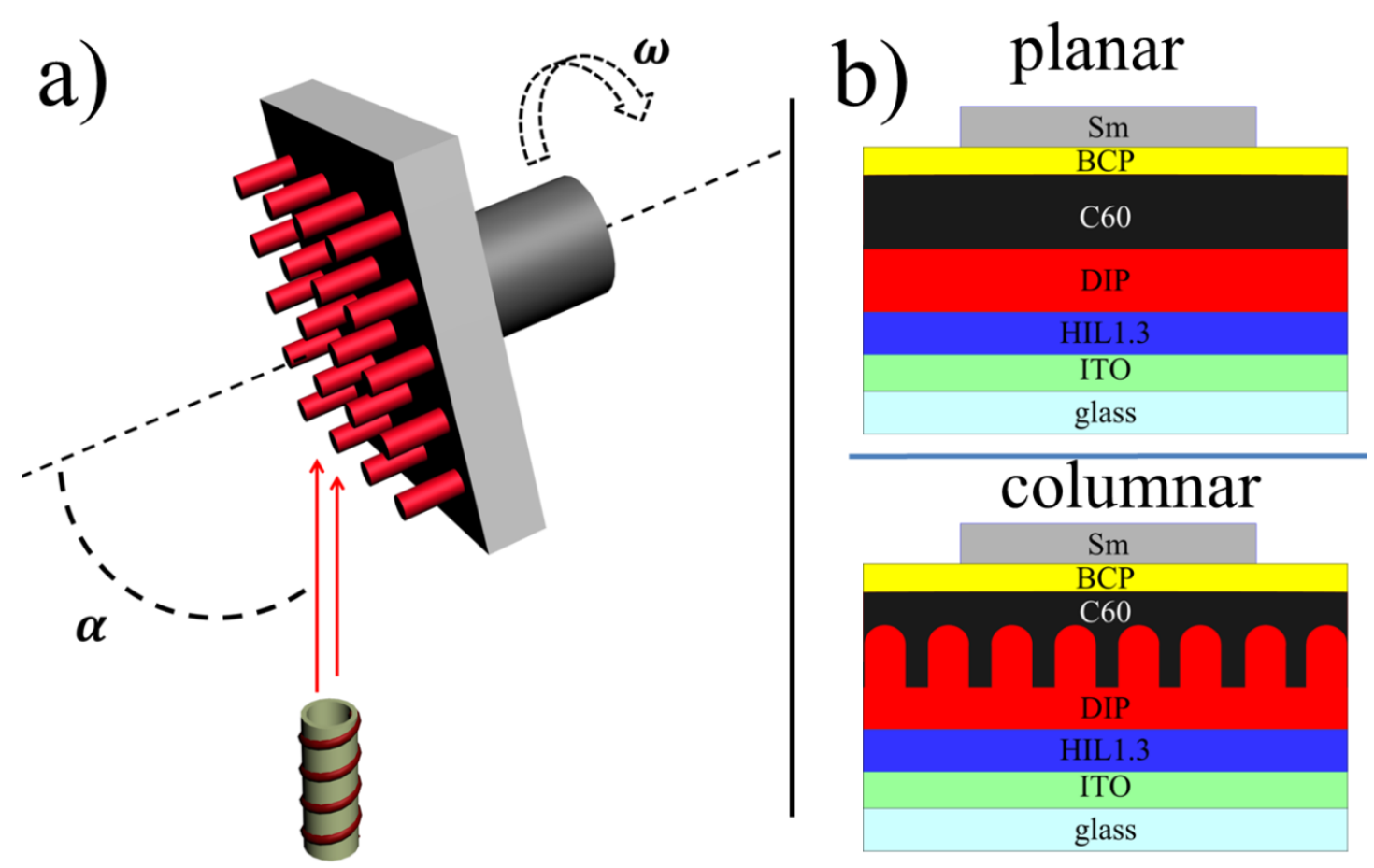

Figure 1: (a) Experimental geometry of Glancing-Angle Deposition (GLAD); $\alpha$ is the angle of the molecular flux with respect to the sample normal and $\omega$ the substrate rotation frequency. (b) Scheme of the OPVC structures used; top: conventional bilayer OPVC, bottom: OPVC with DIP-NCs.
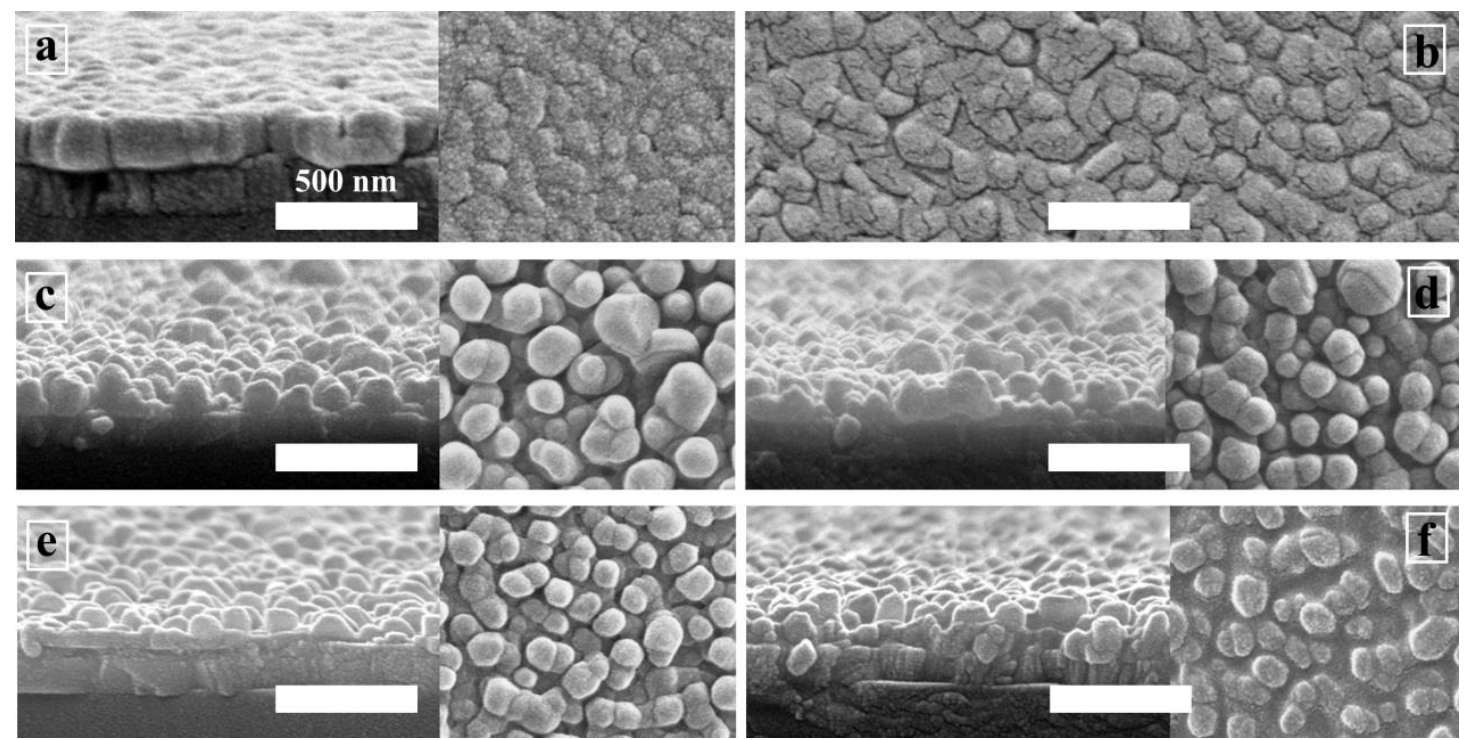

Figure 2: SEM micrographs (cross section and top view) of DIP on bare ITO (left column) and PEDOT:PSS coated ITO (right column). (a-b): $50 \mathrm{~nm}$ vertically deposited DIP films; (c-d): $100 \mathrm{~nm}$ DIP-NCs; (e-f): $100 \mathrm{~nm}$ DIP-NCs on a vertically deposited $10 \mathrm{~nm}$ DIP underlayer. 

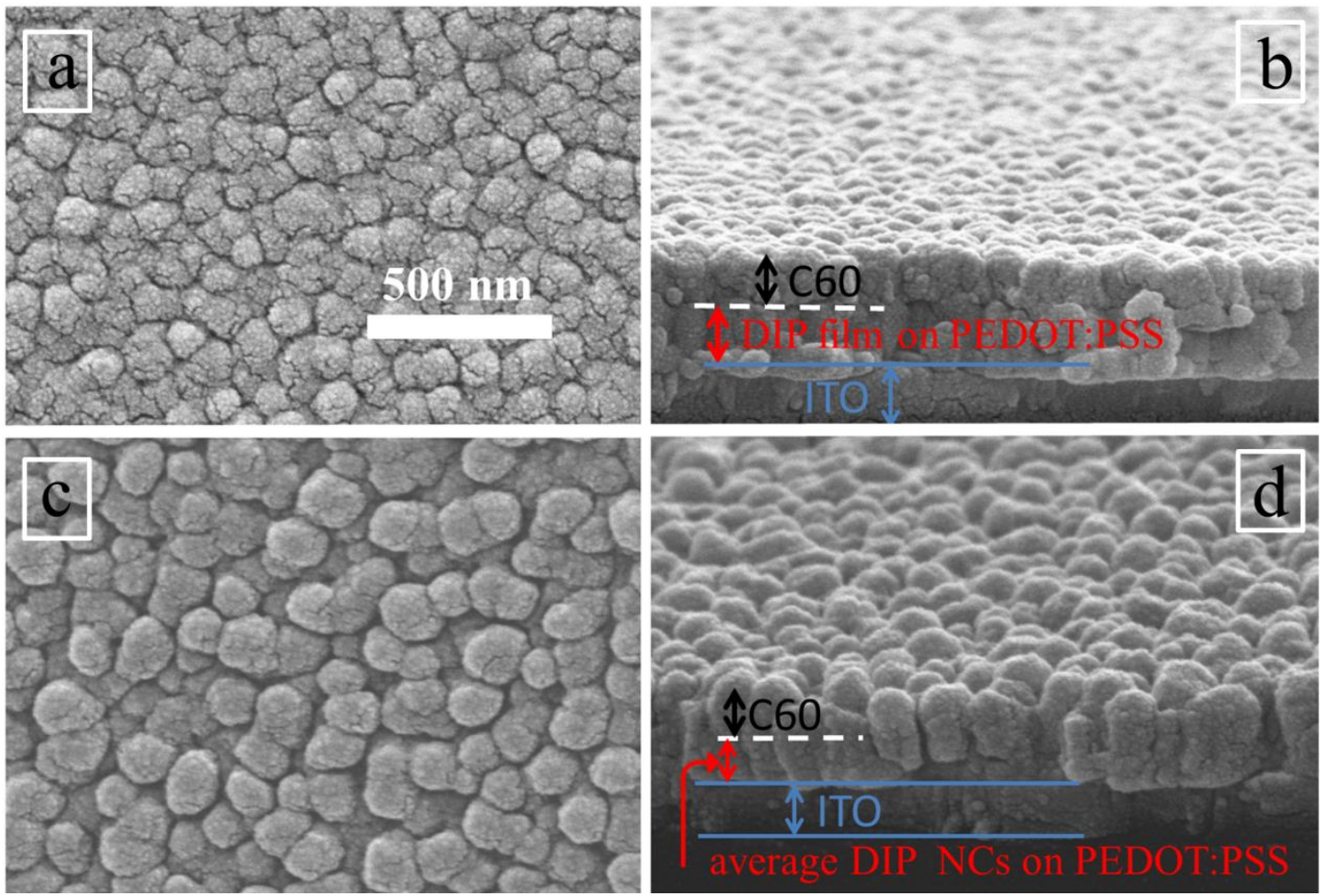

Figure 3: SEM micrographs of the planar C60/DIP heterojunction (a-b), and the C60/DIP-NC heterojunction (c-d), in top view (left) and cross section view (right); average DIP NC height was determined from a sample not coated with $\mathrm{C} 60$, but otherwise prepared identically.

a)

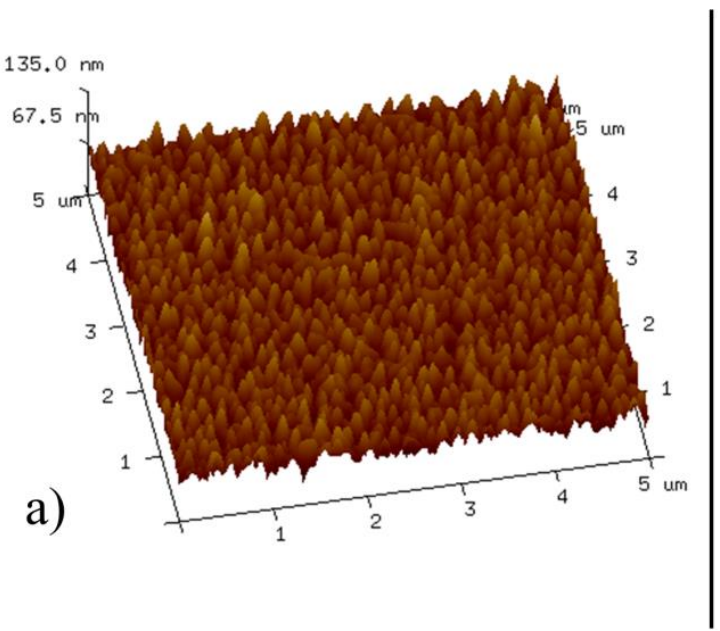

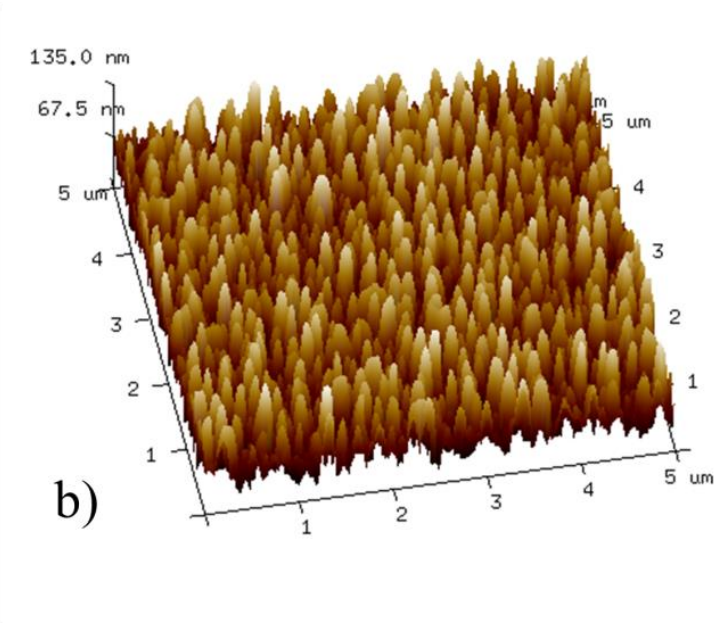

b)

Figure 4: AFM micrographs of (a) a vertically deposited DIP/PEDOT:PSS film (SEM c.f. Fig. 2b), and (b) DIP-NCs/PEDOT:PSS (SEM c.f. Fig. 2d). 

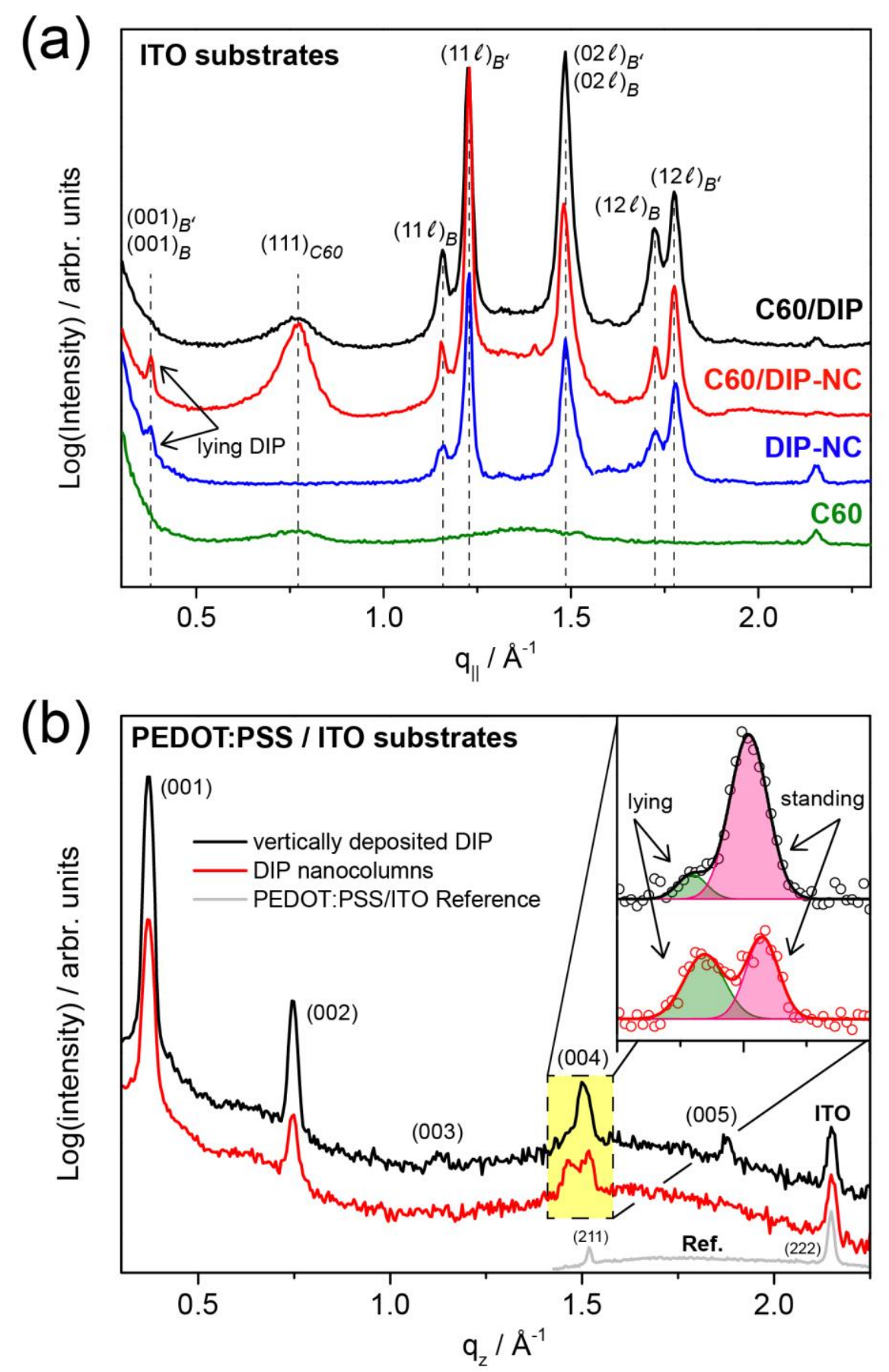

Figure 5: (a) In-plane GIXRD data of nominally $60 \mathrm{~nm}$ C60 on $90 \mathrm{~nm}$ vertically deposited DIP (black curve) and on $100 \mathrm{~nm} \mathrm{C60} \mathrm{on} 160 \mathrm{~nm}$ DIP-NCs (red curve), as well as of reference samples of $160 \mathrm{~nm}$ DIP NCs (blue curve) and $20 \mathrm{~nm} \mathrm{C60} \mathrm{(green} \mathrm{curve);} \mathrm{the} \mathrm{data}$ was extracted from the respective reciprocal space maps (Fig. 6) by integrating along qz (range: $0.0-0.1 \AA-1$ ). All films were deposited on bare ITO substrates. Index labels $B$ and $B$ ' indicate the two different observed crystalline phases of DIP (see text). (b) Specular XRD of nominally $50 \mathrm{~nm}$ vertically deposited DIP (black curve), and on $100 \mathrm{~nm}$ DIP-NCs on a $10 \mathrm{~nm}$ DIP underlayer (red curve), both on PEDOT:PSS coated ITO substrates with a PEDOT:PSS/ITO reference sample (grey curve) with the ITO (222) and (211) peaks observed. 
The inset is a zoom of the (004) reflection highlighting the contribution of lying DIP molecules (" $\lambda$-phase") ${ }^{45}$, which is enhanced in the DIP-NC sample.
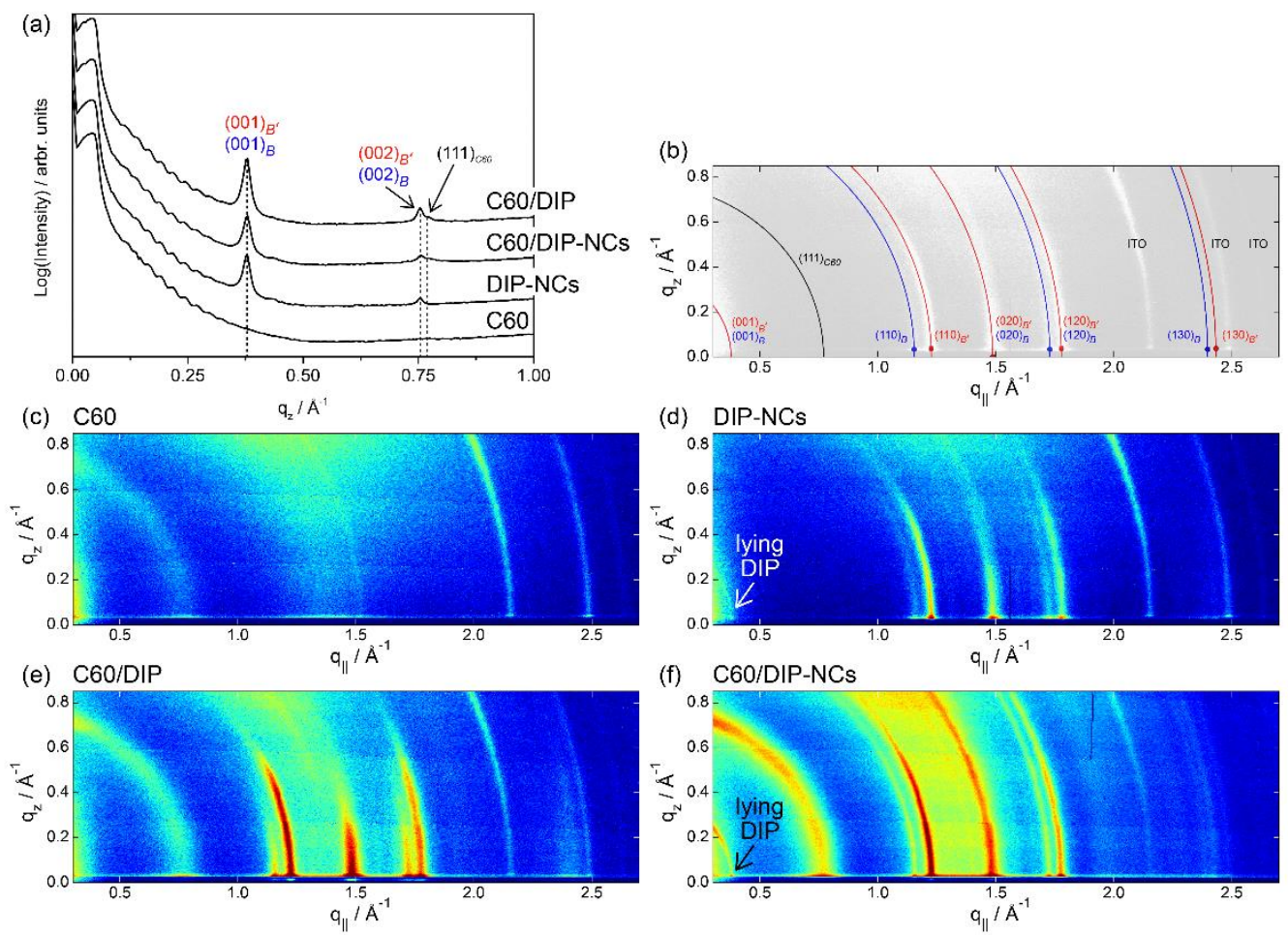

Figure 6: Specular X-ray diffraction (XRD) and grazing-incidence X-ray diffraction (GIXRD) data of the investigated DIP films on bare ITO substrates: (a) XRD data of $60 \mathrm{~nm}$ C60 on $90 \mathrm{~nm}$ vertically deposited DIP [GIXRD: (e)], $100 \mathrm{~nm}$ C60 on nominally $160 \mathrm{~nm}$ thick DIP nanocolumns (DIP-NCs) established by glancing-angle deposition (GLAD) [GIXRD: (f)], as well as pure reference films of $160 \mathrm{~nm}$ DIP-NCs [GIXRD: (d)] and $20 \mathrm{~nm}$ C60; peaks labelled with $B$ and $B$ ' relate to the polymorphs discussed in the text. (b) Indexation of the reflections observed in GIXRD (c-f); from the $q_{\|}$positions, the unit-cell parameters of the DIP polymorphs can be estimated. 

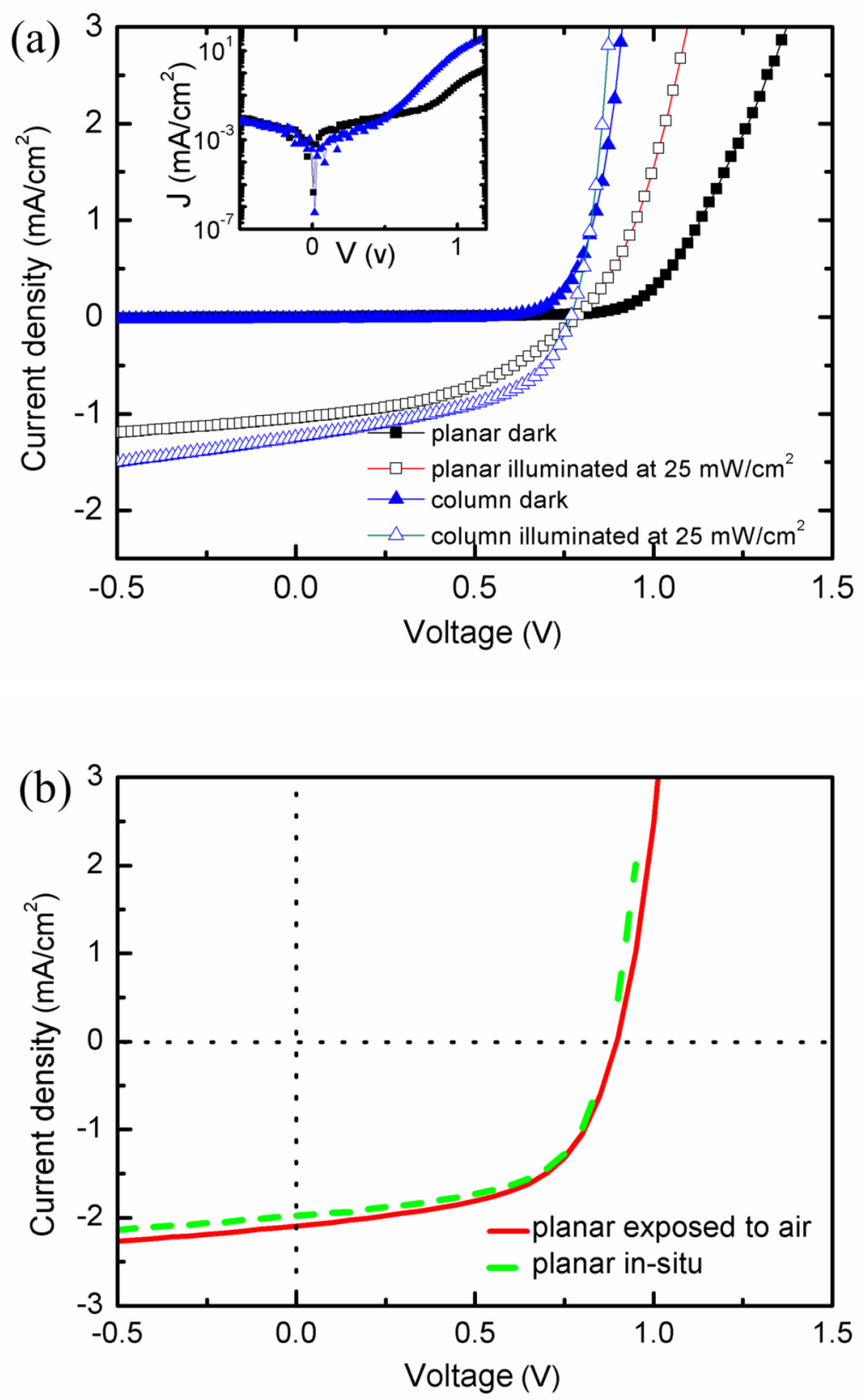

Figure 7: (a) J-V characteristics of bilayer and DIP-NC based OPVCs. Black closed/open square symbols: bilayer device in dark/illuminated $\left(25 \mathrm{~mW} / \mathrm{cm}^{2}\right)$ in vacuum; blue closed/open triangle symbols: DIP-NC based device in dark/illuminated $\left(25 \mathrm{~mW} / \mathrm{cm}^{2}\right)$ in vacuum. Inset: $\mathrm{J}-\mathrm{V}$ curves in dark in logarithmic representation. (b) Comparison of OPVC characteristics of bilayer devices measured in-situ and after exposure to ambient air demonstrating negligible degradation under $100 \mathrm{~mW} / \mathrm{cm}^{2}$ illumination. 


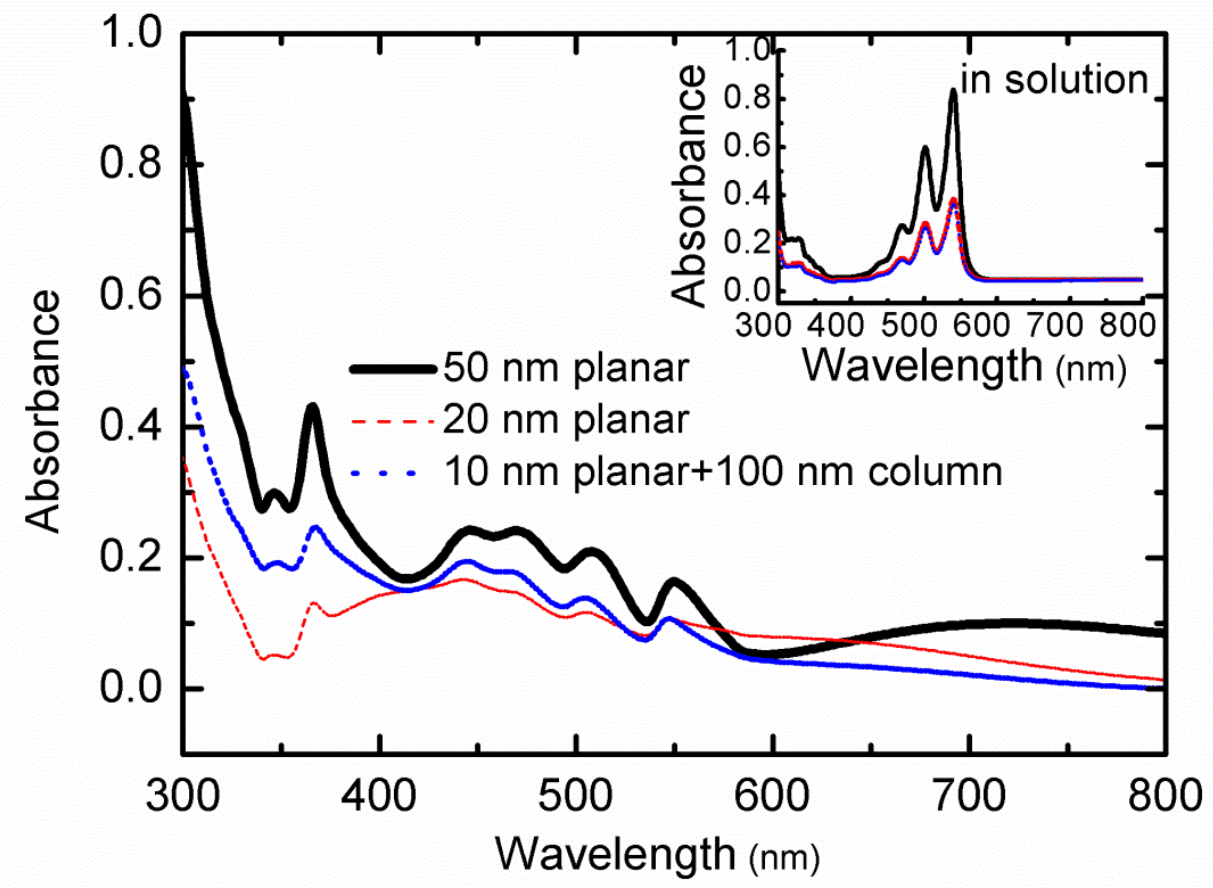

Figure 8: UV-VIS absorption spectra of $50 \mathrm{~nm}$ (black curve) and $20 \mathrm{~nm}$ (red point curve) vertically deposited DIP/PEDOT:PSS, and that of $100 \mathrm{~nm}$ DIP-NCs on a vertically deposited $10 \mathrm{~nm}$ DIP underlayer on PEDOT:PSS (blue dash curve). Inset: UV-VIS absorption spectra of the $50 \mathrm{~nm}$ sample (black curve), $20 \mathrm{~nm}$ sample (red dash curve) and the NC-based sample (blue point curve) dissolved from the substrate in equal amount of solvent and measured in solution. 